\title{
Nephrectomy for Retroperitoneal Sarcoma: Stay Calm and (Cautiously) Carry On
}

\author{
Mark Fairweather, $\mathrm{MD}^{1,2,3}$, and Chandrajit P. Raut, MD, MSc ${ }^{1,2,3}$ \\ ${ }^{1}$ Department of Surgery, Brigham and Women's Hospital, Boston, MA; ${ }^{2}$ Center for Sarcoma and Bone Oncology, Dana- \\ Farber Cancer Institute, Boston; ${ }^{3}$ Harvard Medical School, Boston, MA
}

Soft tissue sarcomas are a rare group of tumors, each with their own inherent biologic behavior driven by histologic subtype and location. There have been significant advancements in recent years in understanding the unique tumor biology of those histologic subtypes arising within the retroperitoneum from studies conducted through multiinstitutional and international collaboration. Surgery, often involving en bloc multivisceral resection, remains the cornerstone treatment for localized retroperitoneal sarcomas (RPS). The extent of resection has been a source of debate over recent years. At a minimum, the extent of resection should be guided by anatomic considerations and histologic-specific tumor biology that ultimately dictates the risk of adjacent organ infiltration, patterns of local/ distant failure, and survival.

The extent of resection must be balanced with the associated risks of surgery. The 30-day postoperative morbidity (Clavien-Dindo grade $\geq 3$ ) has been reported to be $16.4 \%$ in one of the largest series of patients undergoing resection of primary RPS by the Transatlantic Australasian Retroperitoneal Sarcoma Working Group (TARPSWG). ${ }^{1}$ In this study, patterns of resection that included vascular resection or pancreaticoduodenectomy were associated with greater risk of perioperative morbidity. While a nephrectomy may be associated a relatively low risk of morbidities traditionally captured within the Clavien-

(C) Society of Surgical Oncology 2020

First Received: 14 September 2020

Accepted: 15 October 2020;

Published Online: 4 November 2020

M. Fairweather, MD

e-mail: mfairweather@bwh.harvard.edu
Dindo classification, limited data exist to guide management with regards to post-nephrectomy renal specific outcomes.

In this issue of Annals of Surgical Oncology, Stahl and colleagues report post-nephrectomy renal function outcomes in 858 patients undergoing surgery for either primary or recurrent/metastatic RPS from eight institutions as part of the US Sarcoma Collaborative. ${ }^{2}$ Three patients $(0.35 \%)$ required postoperative dialysis, of whom one had undergone nephrectomy $(n=191,0.52 \%)$ while two had not $(n=667,0.30 \%)$. The authors performed a matched cohort analysis that demonstrated that those patients who underwent nephrectomy had significantly higher postoperative peak creatinine values and higher rates of both postoperative acute kidney injury (AKI) and acute renal failure (ARF). Age was found to be predictive of AKI, while nephrectomy was predictive of both AKI and ARF.

The results of this study provide data to support both the authors' hypothesis and the relatively common belief that, despite the paucity of data, nephrectomy is associated with worse postoperative renal function. The risk of postoperative renal dysfunction should be routinely discussed with patients as a theoretical risk as part of the informed consent process when nephrectomy is being considered. This study now provides data that can be shared with patients to give more definitive assessment of the risk of postoperative renal dysfunction. For those patients undergoing nephrectomy within the matched cohort $(n=108)$, the rate of postoperative AKI was $14.8 \%$ and ARF 4.6\%. The need for postoperative dialysis is rare and with rates varying slightly based on denominator being used (i.e., all patients versus only nephrectomy patients), however, are consistently $<1 \%$. Importantly, of the three patients in the study 
who required dialysis, all had estimated glomerular filtration rates $(\mathrm{eGFR}) \leq 44$ (chronic kidney disease (CKD) stage $\geq 3 b$ ).

The data reported in this study are consistent with previous reports from single-institution retrospective studies. Approximately $50 \%$ of patients will develop new CKD stage $\geq 3$ following nephrectomy based on reports from the Royal Marsden Hospital $(n=113),{ }^{3}$ Mayo Clinic Florida $(n=47),{ }^{4}$ and Massachusetts General Hospital $(n=54){ }^{5}$ Only two patients out of the 214 combined patients $(0.93 \%)$ from these studies required postoperative dialysis. Of note, results from the only available prospective cohort of patients undergoing surgery \pm neoadjuvant radiation for primary RPS from the STRASS trial reported postoperative renal dysfunction in 14 of 199 patients (7.0\%) undergoing nephrectomy. ${ }^{6}$

The rates of ARI and ARF reported in the current study are difficult to compare as the authors chose to use the Risk, Injury, Failure, Loss, End-stage renal disease (RIFLE) criteria based on creatinine alone to define these terms as opposed to using eGFR to determine CKD stage. As a result, the incidence of ARI and ARF are binary outcomes based on serum creatinine alone, while eGFR calculation using the commonly used CKD-Epidemiology Collaboration formula allows classification into CKD stage that exists on a spectrum of five categories (stage $1: \geq 90$, 2: 60-89, 3: 30-59, 4: 15-29, 5:<15). ${ }^{7}$ eGFR analysis allows more granular analysis of the trend of postnephrectomy renal function and potential clinical implications of renal dysfunction. Additionally, as opposed to using only serum creatinine for RIFLE criteria, eGFR calculated by the CKD-Epidemiology Collaboration formula factors in the influence of age, gender, and race on glomerular filtration.

One critical point to consider when interpreting the results of studies investigating post-nephrectomy renal function is the timing of the assessment of renal function postoperatively. The deterioration and recovery of renal function is a dynamic process. It could be argued that the overall trend is more important and that it is a more accurate assessment than at a single time point after surgery. In the current study, the authors chose to use the peak creatinine within 90 days postoperatively. Previous studies have compared eGFR values at peak creatinine as well as eGFR at last follow-up to assess the overall trend. The clinical significance of peak creatinine, or eGFR, alone is unclear and lends itself to greater degree of confounding by ongoing clinical situation (i.e., dehydration, iodinated contrast-induced nephropathy).

Additionally, it should be noted that patients with both primary and recurrent disease were included in this study. This confounds the rate of nephrectomy, as patients with recurrent disease may have previously undergone nephrectomy during the primary resection. The distribution of histologic subtype would also differ in those with recurrent disease due to the likely patterns of local and distant failure [i.e. leiomyosarcoma (LMS) distant failure, well-differentiated liposarcoma (WDLPS) local failure]. Histologic subtype can guide the necessity for nephrectomy based on risk of histopathologic organ invasion. Our group has previously reported that the risk of histopathologic organ invasion is isolated to patients with dedifferentiated liposarcoma undergoing nephrectomy, while nephrectomy in those patients with LMS and WDLPS could be potentially avoided as long as there is no resulting tumor capsule violation. ${ }^{8}$

Future studies are needed in order to capture the clinical details of patients with ARI/ARF and those requiring dialysis in order to determine whether deterioration of renal function is occurring as an isolated event directly related to nephrectomy or as part of multisystem organ failure from a primary postoperative complication (i.e. bleed, anastomotic leak, etc.). Additionally, the outcomes for patients with preoperative renal dysfunction (eGFR $<60$, CKD stage $\geq 3$ ) remain unclear. When combining results of the previously mentioned single-institution studies, of the 28 patients with preoperative stage 3 CKD only two patients (7.1\%) developed worsening CKD to stage 4 or $5 .^{3-5}$ Data reported for nephrectomy for renal cell carcinoma have demonstrated the somewhat counterintuitive finding that the likelihood of postoperative eGFR recovery to preoperative baseline was significantly higher in patients with preoperative eGFR $<60$ than in those with preoperative eGFR $\geq 60 .{ }^{9}$ This could be attributed to preoperative initiation of compensatory mechanisms in the setting of preoperative renal dysfunction that can occur commonly secondary to hydronephrosis from ureteral obstruction by the tumor. As a result, the remaining contralateral kidney has already gradually started compensating prior to the nephrectomy and recovery is faster than when the compensatory mechanism is not initiated until after nephrectomy. Further investigation into the role of nuclear renal scans in assessing the presence of compensatory measures preoperatively and ability to predict postoperative renal recovery is merited.

These data provide further clarity for both clinicians and patients regarding the rarity of ARI/ARF that ultimately requires dialysis. Predictors of postoperative renal dysfunction remain elusive. Equally important are predictors of postoperative eGFR recovery to preoperative eGFR. This is in part due to the rarity of these events. The impact of prior nephrectomy and renal function status can significantly influence the management of recurrent disease as some systemic chemotherapy agents (i.e., ifosfamide) can be nephrotoxic. Nephrectomy should be performed when clinically indicated and is appropriate based on assessment 
of comorbidities. The inherent risks of these complex operations should always be balanced with the potential oncologic benefit.

DISCLOSURE The authors declare no conflict of interest.

\section{REFERENCES}

1. MacNeill AJ, Gronchi A, Miceli R, et al. Postoperative morbidity after radical resection of primary retroperitoneal sarcoma: a report from the transatlantic RPS Working Group. Ann Surg. 2018;267(5):959-964.

2. Stahl CS, Schwartz PB, Ethun CG, et al. Renal function after retroperitoneal sarcoma resection with nephrectomy: a matched analysis of the United States Sarcoma Collaborative Database. Ann Surg Oncol. 2020. https://doi.org/10.1245/s10434-020-09290-z.

3. Smith HG, Panchalingam D, Hannay JA, et al. Outcome following resection of retroperitoneal sarcoma. $\mathrm{Br} J$ Surg. 2015;102(13):1698-1709.

4. Kim DB, Gray R, Li Z, Wasif N, Bagaria SP. Effect of nephrectomy for retroperitoneal sarcoma on post-operative renal function. J Surg Oncol. 2018;117(3):425-429.
5. Hull MA, Niemierko A, Haynes AB, et al. Post-operative renal function following nephrectomy as part of en bloc resection of retroperitoneal sarcoma (RPS). J Surg Oncol. 2015;112(1):98-102.

6. Bonvalot S, Gronchi A, Le Pechoux C, et al. Preoperative radiotherapy plus surgery versus surgery alone for patients with primary retroperitoneal sarcoma (EORTC-62092: STRASS): a multicentre, open-label, randomised, phase 3 trial. Lancet Oncol. 2020;21(10):1366-1377.

7. Levey AS, Stevens LA, Schmid $\mathrm{CH}$, et al. A new equation to estimate glomerular filtration rate. Ann Intern Med. 2009;150(9):604-612.

8. Fairweather M, Wang J, Jo VY, Baldini EH, Bertagnolli MM, Raut $\mathrm{CP}$. Surgical management of primary retroperitoneal sarcomas: rationale for selective organ resection. Ann Surg Oncol. 2018;25(1):98-106.

9. Zabor EC, Furberg H, Lee B, et al. Long-term renal function recovery following radical nephrectomy for kidney cancer: results from a multicenter confirmatory study. $J$ Urol. 2018;199(4):921-926.

Publisher's Note Springer Nature remains neutral with regard to jurisdictional claims in published maps and institutional affiliations. 